'Departamento de Ciencias Neurológicas Oriente, Facultad de Medicina, Universidad de Chile. Santiago, Chile.

${ }^{3}$ Servicio de Neurología, Hospita del Salvador, Santiago, Chile ${ }^{3}$ Departamento de Medicina, Universidad de Chile, Sección de Hematología, Servicio de Medicina, Hospital del Salvador. Santiago, Chile.

Financiamiento: Los autores declaran no haber recibido financiamiento para la realización de este trabajo.

Conflicto de intereses: Los autores declaran no tener conflictos de interes.

Recibido el 29 de noviembre de 2017, aceptado el 9 de enero de 2018.

Correspondencia a: Gabriel Cea Departamento de Ciencias Neurológicas Oriente, Facultad de Medicina, Universidad de Chile, Servicio de Neurología, Hospital del Salvador, Santiago, Chile, Avenida Salvador 364, Providencia, Santiago Chile. Fono: 56225753568 jcea@med.uchile.cl

\section{Miastenia gravis y su asociación con trastornos linfoproliferativos: casos clínicos}

GABRIEL CEA $^{1,2}$, ANDRÉS GALLARDO V. ${ }^{1}$, MARÍA ELENA CABRERA C. ${ }^{3}$

\section{Myasthenia gravis and its association with lymphoproliferative disorders: a case series}

Myasthenia gravis (MG) is a rare autoimmune disease of the neuromuscular junction. It is characterized by variable weakness and excessive fatigability of skeletal muscles. In the last few years, numerous reports have been published showing the association between autoimmune diseases, such as systemic erythematous lupus or rheumatoid arthritis, with lymphoid neoplasias. The association between MG and lymphoid neoplasia seems to be less frequent. To analyze this association we reviewed the MG patients in the Department of Neurology, Hospital Salvador of Santiago, Chile. During a three-year period we identified four patients who developed different lymphoproliferative disorders: two with B-cell lymphoma, one with chronic lymphocytic leukaemia and one plasmacytoma with an associated amyloidosis. The MG was generalized but mild, all cases classified as type IIa according to the definition proposed by the MG Foundation of America. The neoplasia appeared two to 36 years after the onset of MG. These cases provide additional evidence of the association between $M G$ and lymphoproliferative disorders.

(Rev Med Chile 2017; 145: 1626-1630)

Key words: Autoimmune Diseases; B Cell; Chile; Chronic B-Cell; Leukemia, Lymphocytic; Lymphoma, B-Cell; Lymphoproliferative disorders; Myasthenia Gravis; Plasmacytoma.

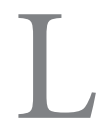

a Miastenia Gravis (MG) es una enfermedad neuromuscular de origen autoinmune, poco frecuente con una prevalencia de $1,5-$ 17,9 por $100.000^{1}$ y una incidencia de $0,3-3$ por $100.000^{2}$, a nivel mundial. La MG es la primera enfermedad descrita mediada por anticuerpos, contra los receptores nicotínicos de acetilcolina $(\mathrm{AChR})^{3}$. La clínica se caracteriza por debilidad variable y fatigabilidad fluctuante de los músculos esqueléticos que puede afectar exclusivamente los ojos, forma ocular, o comprometer la mayoría de los músculos esqueléticos en la forma generalizada de la enfermedad, detectándose anticuerpos contra el AChR en 50\% y 90\%, respectivamente. Los pacientes seronegativos presentan anticuerpos contra otras estructuras relacionadas con el AChR que alteran su estructura o función, como anticuerpos contra la tirosina quinasa muscular $(\mathrm{MuSK})^{4} \mathrm{O}$ contra la proteína 4 relacionada con el receptor de lipoproteína de baja densidad (LRP-4) ${ }^{5}$. Además, en alrededor de 10-15\% la MG se asocia a timoma, como un fenómeno paraneoplásico.

En los últimos años se ha establecido una asociación entre enfermedades autoinmunes, como el lupus eritematoso sistémico (LES), artritis reumatoide (AR) y síndrome de Sjögren (SS), con neoplasia linfoide, por un mecanismo de linfomagénesis aún no del todo precisado ${ }^{6}$. En relación con MG se han reportado alrededor de 50 casos de neoplasia linfoide, lo que sugiere una relación mayor que la simple casualidad ${ }^{7}$. Con el objeto de investigar esta asociación, realizamos una revisión 
de los pacientes con MG (alrededor de 90) atendidos en nuestro hospital e identificamos entre el 2013-2016, cuatro pacientes que desarrollaron una neoplasia linfoproliferativa. A continuación se describe cada caso en forma detallada. Este estudio fue aprobado por el Comité de Ética Científico del Servicio de Salud Metropolitano Oriente.

\section{Caso 1}

Mujer, que en 1990, a los 30 años de edad, presentó purpura trombocitopénico autoinmune, refractaria a corticoides pero que, respondió satisfactoriamente a esplenectomía. Diez y seis años más tarde, en mayo de 2006, consulta por un cuadro de 2 meses de evolución, caracterizado por fatigabilidad y debilidad en actividades de la vida diaria como peinarse y subir escaleras. Al examen, presentaba debilidad en cintura escapular y pelviana. El estudio electrofisiológico fue compatible con un defecto en la transmisión en la unión neuromuscular, la detección de anticuerpos contra AChR, fue de $68,6 \mathrm{nmol} / \mathrm{L}$ (normal $\leq 0,02$ ) y una tomografía computada (TC) de tórax descartó la presencia de timoma. Fue tratada con piridostigmina $60 \mathrm{mg} 5$ veces al día, con buena respuesta clínica, logrando realizar sus actividades cotidianas sin dificultades. Seis años después, estando con buen control de la MG, presentó tos persistente, disnea y baja de peso de $6 \mathrm{Kg}$ en 6 meses. La TC de tórax mostró imagen compatible con bronquiolitis obliterante con neumonía organizante (BOOP), adenopatías en región hiliar izquierda y mediastino, atribuible a proceso linfoproliferativo (Figura 1). Se realizó una biopsia transbronquial que resulta concordante con neoplasia de células plasmáticas, CD20, CD3,CD30 y CD138 positivos, y tinción rojo Congo positiva para amiloidosis. La electroforesis de proteínas no mostró componente monoclonal y la relación Lambda/Kappa fue 0,98 , descartandose amiloidosis sistémica. Se diagnosticó una amiloidosis ganglionar y se inició tratamiento con melfalan y ciclofosfamida, con disminución significativa de la disnea y la obstrucción bronquial. Actualmente se encuentra en buenas condiciones, con discreta obstrucción bronquial, y tratamiento con ciclofosfamida oral $300 \mathrm{mg}$. semanal y la MG en estatus mínimamente sintomático, con piridostigmina $60 \mathrm{mg}$ tres veces al día.

\section{Caso 2}

Mujer que consultó a los 44 años, en mayo 1980 , por cuadro de ptosis palpebral fluctuante y debilidad en extremidades superiores. Se realizó test de edrofonio que resultó positivo, el estudio electrofisiológico fue compatible con defecto de la unión neuromuscular y se practicó mediastinoscopia que descartó timoma. Se inició tratamiento con piridostigmina, con buena respuesta durante un año, momento en que la paciente decide suspender su tratamiento. Como consecuencia, presentó deterioro importante de sus síntomas y fue hospitalizada en nuestro servicio. Sus síntomas fueron controlados con prednisona por un mes y piridostigmina, permaneciendo estable, sólo con piridostigmina $60 \mathrm{mg}$ cuatro veces al día. Se mantuvo estable hasta 2013 cuando, encontrándose con sintomatología mínima tomando piridostigmina $60 \mathrm{mg}$ tres veces al día, un hemograma de control reveló leucocitosis de $54.520 \times \mathrm{mm}^{3}$ con $96 \%$ de linfocitos maduros. El estudio de inmunofenotipo por citometría de flujo, mostró linfocitos B con co-expresión CD5/ CD19, CD20 (+), CD23 (+), CD22 débil, FMC7 (-), restricción y expresión débil de cadena liviana kappa. Se diagnosticó leucemia linfocítica crónica (LLC), estadio Binet A. La paciente se mantiene asintomática, sin necesidad de tratamiento para su LLC y sin molestias miasténicas, con piridostigmina $60 \mathrm{mg}$ tres veces al día.

\section{Caso 3}

Varón que consultó en el año 2000, a los 44 años en un hospital del norte del país, por fatigabilidad de extremidades asociada a ptosis palpebral bilateral y diplopía de predominio vespertino. Se realizó test de edrofonio que resultó positivo y se inició tratamiento con piridostigmina $60 \mathrm{mg}$ tres veces al día, con mejoría clínica parcial. La TC de tórax no mostró evidencia de timoma y los anticuerpos AChR fueron positivos, título $18 \mathrm{nmol} / \mathrm{L}$ (normal $\leq 0,02$ ). Se realizó timectomía y posteriormente se agregó azatioprina $100 \mathrm{mg}$ /día que mantuvo por 14 años. En noviembre 2014 inicia cuadro de dolor abdominal persistente en flanco izquierdo, con episodios de diarrea, anorexia y baja de peso de $15 \mathrm{Kg}$ en 6 meses. Se realizó endoscopia digestiva alta que reveló tumor gástrico sub- 

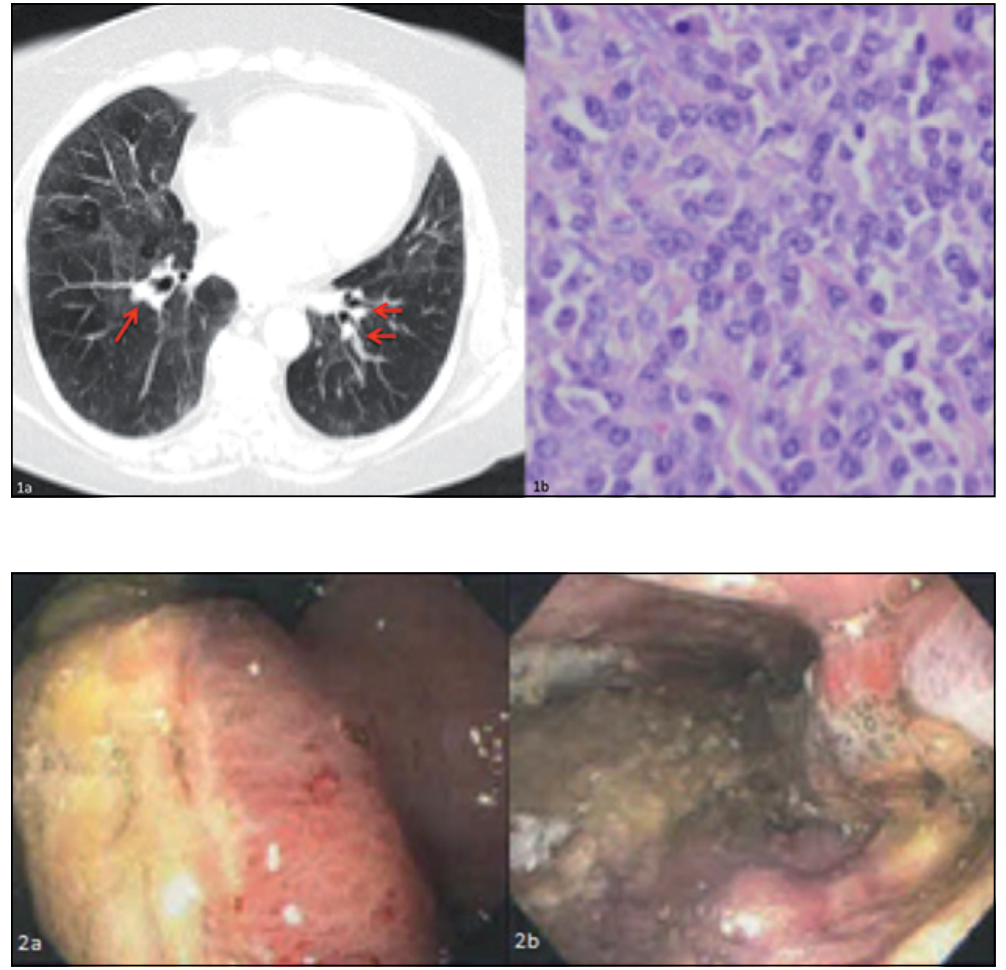

Figura 1. A. TAC de tórax del paciente 1 donde se observan adenopatías perihiliares (flechas rojas), patrón en mosaico e imágenes en vidrio esmerilado, predominio a derecha. B. Biopsia ganglionar del mismo paciente, H\&E de $100 x$, donde se observa proliferación de células plasmocitoide.

Figura 2. Endoscopia digestiva alta correspondiente al caso 3 donde se observa lesión mamelonada con área necróticas, bordes gruesos y congestivos en el fondo gástrico. cardial Bormann III y gastropatía antral crónica atrófica (Figura 2), test de ureasa positiva y biopsia que describió hallazgos compatibles con linfoma no Hodgkin (LNH) difuso de células B grandes (DCGB) etapa clínica IV. Se inició quimioterapia con rituximab, ciclofosfamida, doxorrubicina, vincristina, prednisona (R-CHOP). Sin embargo, presentó múltiples complicaciones infecciosas en contexto de neutropenia febril y progresión del linfoma, falleciendo el 24 julio de 2015.

\section{Caso 4}

Varón de 58 años que, en 2014, se presentó con una historia de 2 años de ptosis palpebral bilateral fluctuante que empeoraba en las tardes, asociada a diplopía, cefaloparesia y debilidad moderada de extremidades de predominio proximal. Se inició tratamiento con piridostigmina y prednisona en dosis bajas, con buena respuesta. La determinación de anticuerpos $\mathrm{AChR}$ fue positiva, título $5,33 \mathrm{nmol} / \mathrm{L}$ (normal $\leq 0,02$ ) y el CT de tórax fue normal. Después de algunos meses suspendió tratamiento, con recurrencia de los síntomas que fueron controlados nuevamente con prednisona $5 \mathrm{mg} /$ día y piridostigmina $60 \mathrm{mg}$ tres veces al día, manteniendo leve ptosis y diplopía que no impide sus actividades diarias. En junio 2016 fue evaluado en hematología por historia de 2 meses de adenopatías cervicales, supraclaviculares y axilares de rápido crecimiento. Se realizó biopsia de ganglio axilar que fue informada como LNH DCGB, etapa IV por compromiso gástrico y óseo. Actualmente en quimioterapia R-CHOP 2 ciclos, con buena respuesta clínica.

\section{Discusión}

Todos los casos presentados corresponden a neoplasias de estirpe B y en todos la MG es de tipo IIa, en la clasificación de la MG Foundation of America, y precedió por 2-36 años, al diagnóstico de neoplasia linfoide. Ningún caso requirió de corticoides en dosis altas o por largos periodos de tiempo o inmunomodulación. Tres nunca recibieron inmunosupresores y el caso 3 tomó azatioprina por un largo periodo pero, en dosis más bajas de lo indicado. 
El caso 1 es una paciente que presenta dos patologías autoinmunes, un purpura trombocitopenico y una $\mathrm{MG}$ con un comportamiento benigno por muchos años, tal como ha sido descrito y que, posteriormente presenta una neoplasia plasmocelular con amiloidosis ganglionar. Las neoplasias plasmocelulares asociadas a MG se han reportado muy ocasionalmente ${ }^{8}$. Pero, es difícil explicar la asociación entre dos patologías autoinmunes poco comunes, y una neoplasia plasmocelular, aún más infrecuente, sin plantearse un mecanismo etiopatogénico común. El caso 2 representa la asociación de MG y LLC, esta última, ampliamente descrita en otras enfermedades autoinmunes ${ }^{9}$. En cambio, se ha comunicado solo 10 casos de coexistencia de MG y LLC, en algunos de los cuales el diagnóstico de MG precedió por años el de la enfermedad oncológica $^{10,11,12}$, con una buena respuesta sintomática a los anticolinesterásicos en la mayoría de los casos, al igual que nuestra paciente que, uso prednisona solo por algunas semanas en un periodo de exacerbación de sus síntomas y nunca fue necesario otro inmunosupresor. En los casos 3 y 4 la MG está asociada a LNH DCGB, que es el subtipo más frecuente asociado a enfermedades autoinmunes como AR, LES y SS ${ }^{13}$ y también a $\mathrm{MG}^{14,15,16}$.

En nuestros pacientes la patología neuromuscular precedió por 2 a 36 años a la neoplasia hematológica. Todos los pacientes presentaron una MG tipo IIa con serología positiva para anticuerpos AChR, excepto una donde el test no se realizó, los pacientes requieren pocos medicamentos para mantener una vida prácticamente normal, sin exacerbaciones que requirieran intervenciones especiales. El trastorno linfoproliferativo aparece muchos años después del inicio de la MG en nuestros casos, por lo que resulta difícil asociarlo con un fenómeno paraneoplásico. Se ha postulado que los factores que gatillan la transición de un proceso autoinmune a una neoplasia linfoproliferativa, probablemente estén relacionados con una estimulación antigénica crónica, que produce una proliferación linfocítica sostenida ${ }^{13,17}$. La consecuencia de este proceso de varios pasos, es el crecimiento desordenado de células B y la alteración de la función de linfocitos $\mathrm{T}^{13}$. En pacientes con $\mathrm{MG}$ se ha descrito además que el factor activador de células B de la familia TNF (BAFF por sus siglas en inglés) a nivel sérico, está elevado, comparado con controles normales ${ }^{18}$. BAFF aumenta la sobrevida de las células B, tanto normales como autoproliferativas, estando altamente asociado a neoplasias de células $\mathrm{B}^{19} \mathrm{y}$ en menor medida como co-estimulador en la activación de células $\mathrm{T}^{20}$. Sin embargo, la sobre-expresión de BAFF no es específico de la MG, ya que también está sobre-expresado en el síndrome de Sjögren, artritis reumatoide y lupus eritematoso sistémico ${ }^{13}$.

\section{Conclusión}

En los últimos años se ha establecido una asociación entre enfermedades autoinmunes, como el lupus eritematoso sistémico, artritis reumatoide y síndrome de Sjögren, con neoplasia linfoide, por un mecanismo de linfomagénesis aún no del todo precisado. Esta relación también se ha planteado con MG y se han publicado alrededor de 50 casos de neoplasia linfoide en la literatura internacional, lo que sugiere una relación mayor que la simple casualidad. Los casos descritos, agregan evidencia sobre una asociación entre MG y neoplasias linfoproliferativas, probablemente por un mecanismo no-paraneoplásico, como ocurre en otras enfermedades autoinmunes crónicas

\section{Referencias}

1. Carr AS, Cardwell CR, McCarron PO, McConville J. A systematic review of population based epidemiological studies in Myasthenia Gravis. BMC Neurol 2010; 8: 10 46.

2. McGrogan A, Sneddon S, de Vries CS. The incidence of myasthenia gravis: a systematic literature review. Neuroepidemiology 2010; 34: 171-83.

3. Vincent A. Unravelling the pathogenensis of myasthenia gravis. Nature Reviews Immunology 2002; 2: 779-804.

4. Evoli A, Tonali PA, Padua L, Monaco ML, Scuderi F, Batocchi AP, et al. Clinical correlates with anti-MuSK antibodies in generalized seronegative myasthenia gravis. Brain 2003; 126: 2304-11.

5. Higuchi O, Hamuro J, Motomura M, Yamanashi Y. Autoantibodies to low-density lipoprotein receptor-related protein 4 in myasthenia gravis. Annals of Neurology 2011; 69: 418-22.

6. Zintzaras E, Voulgarelis M, Moutsopoulos HM. The Risk of Lymphoma Development in Autoimmune Diseases: A Meta-analysis. Arch. Intern Med 2005; 165: 2337-44.

7. Rezania K, Soliven B, Baron J, Lin H, Penumalli V, van 
Besien K. Myasthenia gravis, an autoimmune manifestation of lymphoma and lymphoproliferative disorders: case reports and review of literature. Leuk Lymphoma 2012; 53: 371-80.

8. Bartoloni C, Scoppetta C, Flamini G, Guidi L, Bartoccioni E, Lambiase $\mathrm{M}$, et al. Waldenstrom's macroglobulinemia and myasthenia gravis. J Clin Lab Immunol 1981; 6: 275-8.

9. Kipps TJ, Carson DA. Autoantibodies in chronic lymphocytic leukemia and related systemic autoimmune diseases. Blood 1993; 81: 2475-87.

10. Vogel JM, Kornfeld P, Forte FA, Jones RA, Genkins G, Papatestas AE, et al. Myasthenia gravis: association with chronic lymphocytic leukemia. N Y State J Med 1977; 77: 2252-6.

11. Fujimaki K, Takasaki H, Koharazawa H, Takabayashi M, Yamaji S, Baba Y, et al. Idiopathic thrombocytopenic purpura and myasthenia gravis after fludarabine treatment for chronic lymphocytic leukemia. Leuk Lymphoma 2005; 46: 1101-2.

12. Cohen SM, Waxman S. Myasthenia gravis, chronic lymphocytic leukemia, and autoimmune hemolytic anemia. A spectrum of thymic abnormalities? Arch Intern Med 1967; 120: 717-20.

13. Goldin LR and Landgren O. Autoimmunity and Lymphomagenesis. Int J Cancer. 2009;124(7):1497-1502.
14. Davis S, Schumacher MJ. Myasthenia gravis and lymphoma. A clinical and immunological association. JAMA 1979; 242: 2096-7.

15. Bowen JD, Kidd P. Myasthenia gravis associated with T helper cell lymphoma. Neurology 1987; 37: 1405-8.

16. Manckoundia P, Martin-Pfitzenmeyer I, Pfitzenmeyer P. Myasthenia gravis associated with a B-cell, nonHodgkin's lymphoma. Eur J Intern Med 2006; 17: 146.

17. Strauchen JA. Indolent T-lymphoblastic proliferation: report of a case with an 11-year history and association with myasthenia gravis. Am J Surg Pathol. 2001;25:4115.

18. Kim JY, Yang Y, Moon JS, Lee EY, So SH, Lee HS, et al. Serum BAFF expression in patients with myasthenia gravis. J Neuroimmunology 2008; 199: 151-4.

19. Kuo SH, Yeh PY, Chen LT, Wu MS, Lin CW, Yeh KH, et al. Overexpression of B cell-activating factor of TNF family (BAFF) is associated with Helicobacter pylori-independent growth of gastric diffuse large B-cell lymphoma with histologic evidence of MALT lymphoma. Blood 2008; 112: 2927-34.

20. Sutherland AP, Ng LG, Fletcher CA, Shum B, Newton RA, Grey ST, et al. BAFF augments certain Th1-associated inflammatory responses. J Immunology 2005; 174: 5537-44. 